\title{
Leishmaniasis transmission: distribution and coarse-resolution ecology of two vectors and two parasites in Egypt
}

\author{
Abdallah M. Samy ${ }^{[1],[2], ~ L i n d s a y ~ P . ~ C a m p b e l l ~}{ }^{[1]}$ and A. Townsend Peterson ${ }^{[1]}$
}

[1]. Department of Ecology and Evolutionary Biology, Biodiversity Institute, University of Kansas, Lawrence, Kansas, USA, [2]. Entomology Department, Ain Shams University, Abbassia, Cairo, Egypt.

\begin{abstract}
Introduction: In past decades, leishmaniasis burden has been low across Egypt; however, changing environment and land use has placed several parts of the country at risk. As a consequence, leishmaniasis has become a particularly difficult health problem, both for local inhabitants and for multinational military personnel. Methods: To evaluate coarse-resolution aspects of the ecology of leishmaniasis transmission, collection records for sandflies and Leishmania species were obtained from diverse sources. To characterize environmental variation across the country, we used multitemporal Land Surface Temperature (LST) and Normalized Difference Vegetation Index (NDVI) data from the Moderate Resolution Imaging Spectroradiometer (MODIS) for 2005-2011. Ecological niche models were generated using MaxEnt, and results were analyzed using background similarity tests to assess whether associations among vectors and parasites (i.e., niche similarity) can be detected across broad geographic regions. Results: We found niche similarity only between one vector species and its corresponding parasite species (i.e., Phlebotomus papatasi with Leishmania major), suggesting that geographic ranges of zoonotic cutaneous leishmaniasis and its potential vector may overlap, but under distinct environmental associations. Other associations (e.g., P. sergenti with L. major) were not supported. Mapping suitable areas for each species suggested that northeastern Egypt is particularly at risk because both parasites have potential to circulate. Conclusions: Ecological niche modeling approaches can be used as a firstpass assessment of vector-parasite interactions, offering useful insights into constraints on the geography of transmission patterns of leishmaniasis.
\end{abstract}

Keywords: Leishmania. Sandfly. Ecological niche modeling. Sinai. Egypt. Niche similarity.

\section{INTRODUCTION}

Cutaneous leishmaniasis (CL) is a neglected disease occurring in northern and eastern parts of Egypt. Two parasites are responsible for CL circulation in Egypt: Leishmania major and Leishmania tropica ${ }^{1}$. L. major is responsible for zoonotic cutaneous leishmaniasis (ZCL) transmission in several countries of North Africa, whereas L. tropica is responsible for anthroponotic cutaneous leishmaniasis (ACL) transmission across the Old World ${ }^{2,3}$. Although several reservoirs are associated with CL transmission in different regions, vectors for these parasites are well characterized across the Middle East and Africa: specifically, Phlebotomus papatasi appears to be the potential vector for ZCL and $P$. sergenti for ACL ${ }^{1,4-6}$.

CL transmission is known to respond to climate and land use: for instance, invasion of periurban habitats in South America has been attributed to combinations of changing climates and

Address to: Abdallah M. Samy. Biodiversity Institute/University of Kansas. Lawrence, Kansas, USA.

Phone: 1785 864-3926; Fax: 1785 864-5335

e-mail: samy@ku.edu

Received 18 September 2013

Accepted 29 January 2014 increased habitat perturbation ${ }^{7-10}$. Recent CL epidemics have been reported in Iran and Colombia ${ }^{11-12}$, likely reflecting responses of CL to environmental, demographic, and human behavioral factors ${ }^{13-14}$. Detailed epidemiological studies, with detailed forecasting exercises, are thus needed to understand risk factors and transmission patterns to inform optimum control strategies.

Disease transmission systems comprise suites of interacting organisms (in this case, mammal hosts, Leishmania parasites, and a sandfly vector). All components of these systems are required to permit long-term circulation in a region ${ }^{15}$. New approaches to understanding geographic distributions of species have been developed in biodiversity science ${ }^{15}$ that can be adapted to inform spatial epidemiology. A particularly important advance in this regard has been incorporation of environmental dimensions into analyses of geographic distributions of species ${ }^{16}$, in this case, the interacting species that drive transmission. These dimensions collectively describe coarse-resolution aspects of ecological niches of species, defined as the suite of environmental conditions under which the species can maintain populations without immigrational subsidy ${ }^{17-18}$. Too often, however, techniques in spatial epidemiology ignore environmental dimensions entirely ${ }^{18}$, so incorporation of these new approaches in spatial epidemiology has much to offer to the field.

Studies of CL in Egypt are relevant and important for three reasons: 1) Egypt presents distinct environmental, geographic, and demographic contexts compared to other countries that 
are more frequently studied; 2) Egypt represents a regional 'crossroads,' and thus is a potential source of infection from and to neighboring countries; and 3) Egypt has experienced extreme weather events in recent years (e.g., heavy rains and floods in Sinai and Aswan in 2010 ${ }^{19}$, changing potential risks of disease transmission by changing habitat structure for rodent reservoirs and vector populations; for example, P. sergenti was limited to the South Sinai, but the species subsequently spread to the North Sinai district as well ${ }^{20}$. This paper adds to the picture by illuminating the ecology and geography of leishmaniasis transmission across broad geographic extents. We used ENM approaches to assess niche overlap for vector-parasite interactions, which in turn offers useful insight into constraints on the geography of CL transmission.

\section{METHODS}

\section{Accessible area}

We estimated the accessible area (M) as described in detail by Barve and others ${ }^{21}$ for each species. Our estimates were based on the idea that the four species have broad geographic distributions. Phlebotomus (Phlebotomus) papatasi (Scopoli) is a widely distributed species, occurring from the Atlantic Ocean in the west to eastern India in the east ${ }^{22}$. Phlebotomus (Paraphlebotomus) sergenti (Parrot) is distributed in southern Europe, North Africa, western Asia, central Asia, and northern India $^{22}$.Leishmania major is known to occur across southwestern and central Asia, and in North Africa ${ }^{2}$. Leishmania tropica is widely distributed in eastern and northern Africa, the Middle East, and large parts of $\mathrm{Asia}^{2}$. We thus constrained the study area to Egypt because it is the subset of $M$ where we had access to reliable sampling data for vectors and parasites ${ }^{15}$.

\section{Occurrence data and environmental data}

Occurrence records for Leishmania parasites and their vectors were obtained from our own field records; Research and Training Center on Vectors of Diseases, Ain Shams University, Egypt. All collections were georeferenced originally using Magellan eXplorist 210 GPS units (Magellan System Co., San Dimas, CA). When geographic references were textual in nature, we assigned latitude-longitude coordinates via reference to electronic gazetteers (e.g., http://www.fallingrain.com; see Wieczorek and others ${ }^{23}$ ).

To characterize environmental variation across the country, we used 8-day composite Land Surface Temperature (LST) and monthly Normalized Difference Vegetation Index (NDVI) data from the Moderate Resolution Imaging Spectroradiometer (MODIS) satellite imagery (1 km spatial resolution) for January 2005 to December 2011, downloaded from the Land Processes Distributed Active Archive Center using the NASA Reverb Echo data portal (http://reverb.echo.nasa.gov/reverb). No data values resulting from cloud coverage were interpolated temporally and spatially using a linear function in Java (H. Qiao, pers. comm.). Minimum, maximum, median, and range values were calculated for each grid cell across the entire time period for each data set (LST, NDVI). These same composites were generated for three seasonal time periods: January-March, April-July, and August-December. Minimum and maximum values were calculated as the mean of the top $10 \%$ and bottom $10 \%$ of data values, to reduce influence of potentially spurious values. We performed a principal components analysis on the resulting data sets in R (R Core Team 2013), centering and standardizing the data. A total of $95 \%$ of the cumulative proportion of variance in the data set was explained by the first 19 principal components, which we included as environmental variables in ecological niche modeling calibration.

\section{Ecological niche modeling}

We used ecological niche modeling to relate known occurrences to raster environmental data in an evolutionarycomputing environment to produce a 'model' of the ecological requirements of the species ${ }^{15}$. We used the maximum entropy approach implemented in MaxEnt ${ }^{24}$ to estimate ecological niches for the two sandflies and the two Leishmania parasite species. To compare ecological niches among the species, taking into account the mobility constraint area described above, we calculated Hellinger's $I$ index in R for all pairwise combinations of the four species, to test the null hypothesis of niche similarity between pairs against a 'background' of Egypt, as described above. We excluded L. tropica from comparisons owing to low sample sizes for this parasite in Egypt: it has been reported only once in one of the most remote areas, on the EgyptianPalestinian border ${ }^{1}$. We generated points randomly from across the accessible area equal to numbers of real occurrence data available for each species, with 100 replicate samples. We used these background points to generate niche models in MaxEnt; outputs were converted to binary maps using a $10 \%$ threshold ${ }^{25}$. The null hypothesis of niche similarity was rejected if the $I$ value for comparison of the two niche models for the two species in question fell below the $5^{\text {th }}$ percentile in the random-replicate distribution ${ }^{26}$.

\section{RESULTS}

We assembled a total of 169 records for the two Leishmania parasites and their two vector species across Egypt (Figure 1). Sampling efforts for both sandflies and parasites were focused in the northeastern parts of the country, in the Sinai Peninsula, but some sampling extended country-wide. The sandfly species most commonly recorded was $P$. papatasi $(N=121)$ followed by $P$. sergenti $(N=26)$. Records of $L$. major were obtained from 19 localities, all in northern Sinai; only three records were available for L. tropica, all from El Barth, a community on the EgyptPalestine border ${ }^{1}$, thus constituting a single unique occurrence.

Niche model outputs in the form of maps of environmental suitability for the sandfly vectors and Leishmania parasites in Egypt are shown in Figure 1. Highest suitability for P. papatasi was in the Sinai Peninsula, along the Nile River and across its delta, and in western Egypt. In contrast, model outputs suggested higher suitability for $P$. sergenti across southern Sinai, Upper Egypt, and the western deserts. Model outputs for the parasite L. major suggested highly suitable areas in the Sinai Peninsula, 


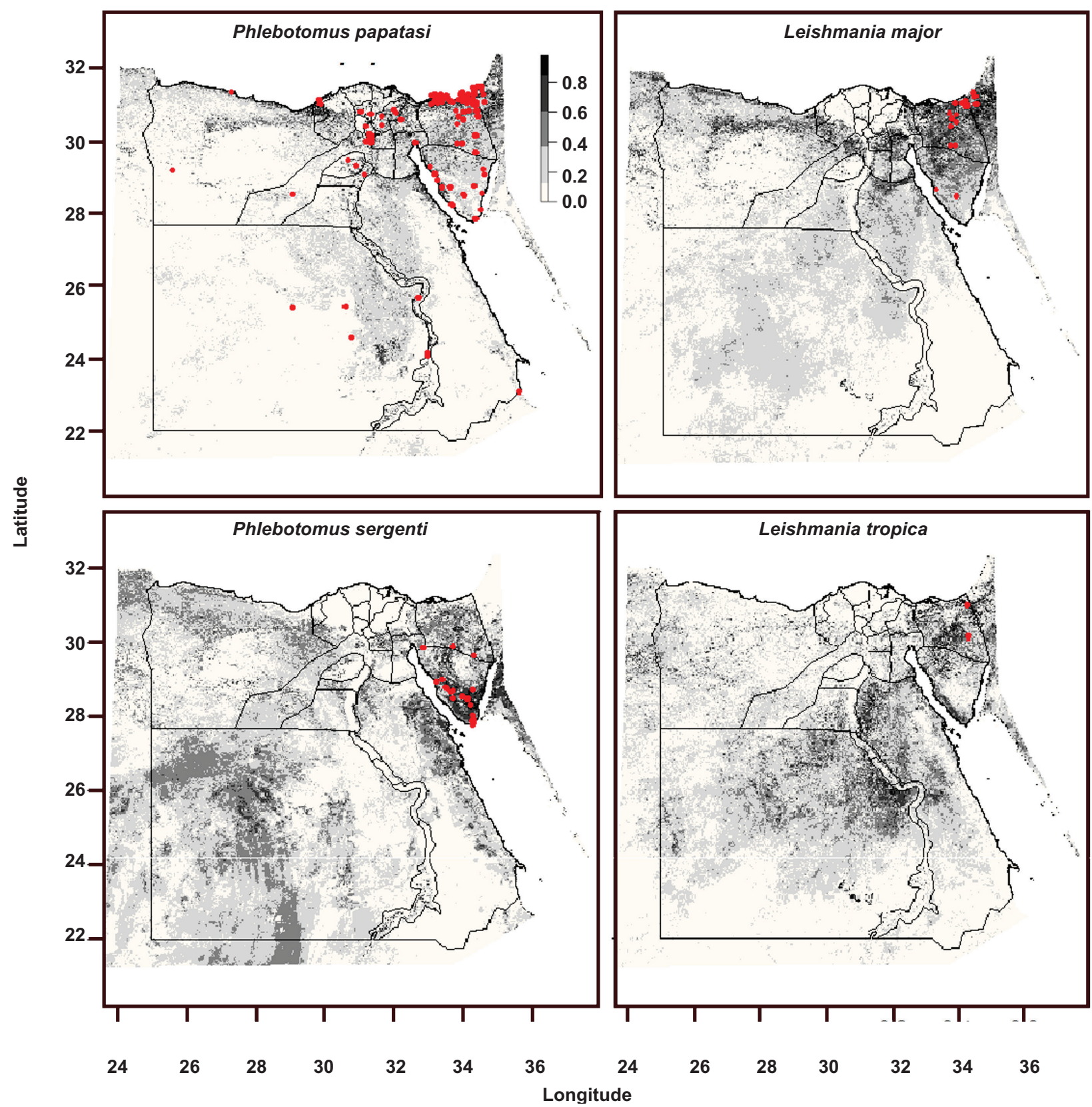

FIGURE 1 - Ecological niche models developed using MaxEnt showing environmental suitability for two vector species and two parasite species associated with cutaneous leishmaniasis transmission in Egypt.

Upper Egypt along the border with Sudan, and on the northern coast bordering Libya. Model outputs for L. tropica were not evaluated, as sample sizes were too small for robust model development; however, its potential vector, $P$. sergenti, occurs in the northeastern Sinai, and was recently found to harbor the parasite ${ }^{1}$.

Phlebotomus papatasi and L. major shared predicted suitable habitat in most areas, except in the southern Sinai and western deserts. We tested the hypothesis of niche similarity for both combinations of the two vector species with $L$. major, and between the two vector species. We rejected the null hypothesis of niche similarity between $P$. papatasi and $P$. sergenti and between L. major and $P$. sergenti. However, we could not reject the null hypothesis of niche similarity between $L$. major and $P$. papatasi (Figure 2).

Assessing where particular vector-parasite combinations have the potential to coincide across Egypt (Figure 3) revealed that highest potential for range overlap between $P$. sergenti and L. major was along the northern coast, in the central region, and in the western deserts of Egypt. However, range overlap between these species was not predicted in current diseaseendemic regions ${ }^{1}$. In contrast, range overlap between P. papatasi 

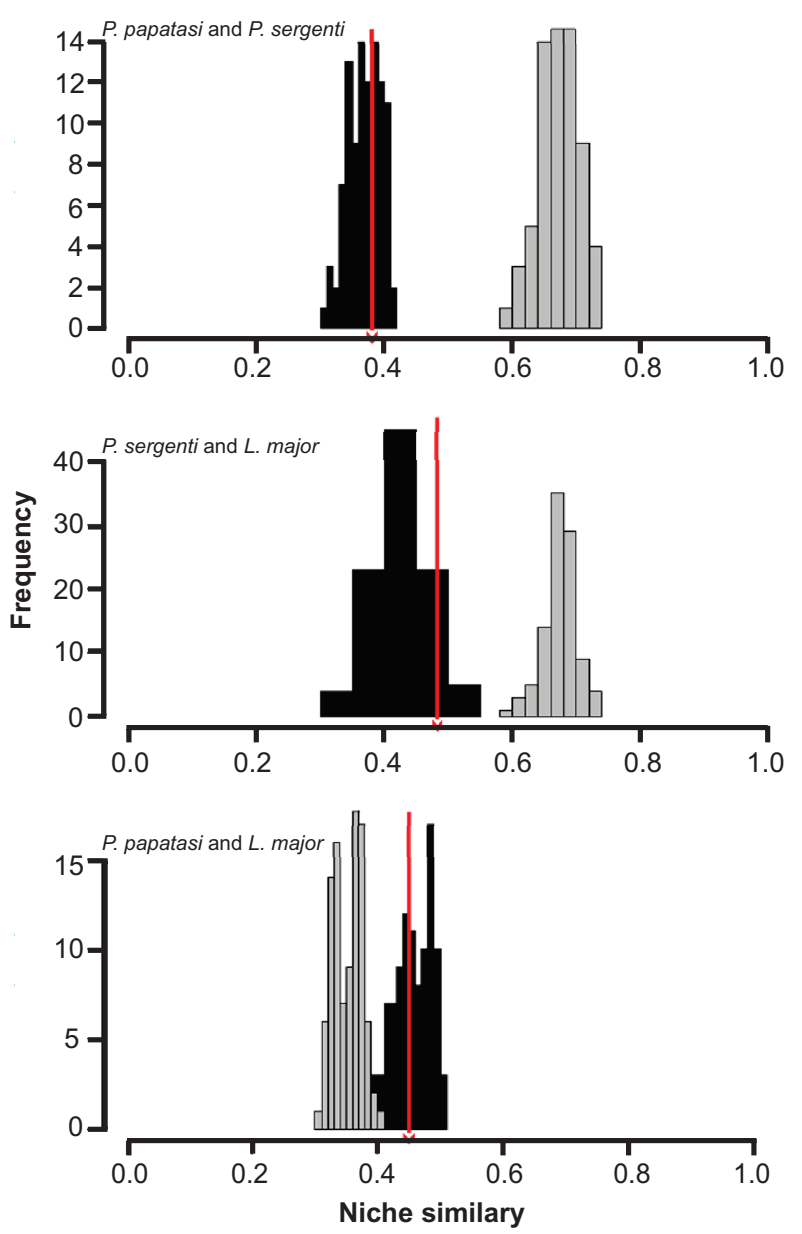

FIGURE 2 - Niche overlap values in background similarity tests based on Hellinger's $I$ similarity statistics. Observed values are shown as a red arrow; null distribution is shown as a histogram. P: Phlebotomus. L: Leishmania.

and L. major existed in northern and central Sinai, and in the northwest of Egypt. Suitable environments for L. major and L. tropica were identified in the northern and central Sinai, Suez Canal, central Egypt, and in narrow zones in the southern and western parts of the country overlapping the single site where L. tropica has been found.

\section{DISCUSSION}

The primary goal of this study was to illuminate details of the ecology and biogeography of $\mathrm{CL}$ and its vectors across broad extents. Geographic and ecological studies of disease vectors and human disease incidence offer powerful approaches for identifying regions potentially at risk for infection, and for exploring potential interactions among hosts, vectors, and parasites ${ }^{27}{ }^{29}$. We used ecological niche modeling and background similarity tests to assess the hypothesis of niche similarity between two vector species ( $P$. papatasi and $P$. sergenti) and one parasite species (L. major) identified in previous studies in the northern Sinai ${ }^{1,4-5}$. Additionally, we mapped range overlap between vector and parasite species, identifying potential areas of co-distribution; such studies have the potential to offer information on species interactions in the $\mathrm{CL}$ transmission cycle that may contribute to human disease risk across Egypt. However, much more detail and consideration of other risk factors (e.g., human socioeconomic variables), are required for full development of risk maps.

In Egypt, most areas affected by leishmaniasis are in desert situations with little vegetation coverage. The Sinai has geographic and demographic attributes making it a crossroads with neighboring countries and territories such as Palestine, Israel, and Jordan, where several Leishmania parasites are endemic ${ }^{30-31}$. The distribution of CL in the Sinai is in low-lying, arid and semiarid deserts for L. major occurrences; however, the few known $L$. tropica occurrences are from suburbs and villages ${ }^{32-33}$. The Sinai presents different habitats in which human populations are scattered across the desert (e.g., Bedouins), and in villages where people from different parts of Egypt work or live. These human activities, along with potential changes in local weather and climate, may create new risk factors for CL distribution in Egypt.

Vector-borne and zoonotic disease transmission risk is the result of interactions between different species in space and time. For example, in the case of leishmaniasis transmission, risk exists when vectors, hosts, and Leishmania parasites co-occur. Here, we mapped disease vectors and Leishmania parasites to identify areas where vector and parasite ranges intersect; we found niche similarity between vectors and parasites that are known to interact (P. papatasi and L. major), and rejected similarity in other cases (P. sergenti and L. major; P. papatasi and P. sergenti). To the authors' knowledge, this study is the first attempt to compare niche similarity between two leishmanisis vector and parasite species in Egypt. The current study has the potential to offer information on species interactions within the $\mathrm{CL}$ transmission cycle that may contribute to human disease risk across Egypt.

Several hypotheses still need to be tested following the first documented emergence of $L$. tropica cases in northern Sinai $^{1}$, particularly when information regarding circulation of this parasite elsewhere in the country, and whether its status is endemic or imported, remains unknown. The possibility exists that $L$. tropica occurrence has been masked by misidentification of the parasite or by taxonomic problems. Although sampling for L. tropica has been carried out in several neighboring countries ${ }^{6,34}$, a major challenge to developing robust ecological and biogeographic hypotheses across Egypt is lack of sampling in key areas. Our results suggested that northeastern portion of the country may be at higher risk than the rest of Egypt, owing to coincidence of suitable areas for both vector and parasite species. Interestingly, mapping predicted potential distributions of the four species together identified areas with potential for coexistence of both transmission cycles; such co-occurrence has been documented in previous studies in Egypt and neighboring countries ${ }^{1,34}$. Clearly, incorporating details of sandfly ecology and distributions in relation to human cases distribution and environmental conditions represents a significant challenge as a next step toward a synthetic understanding of leishmaniasis transmission patterns. 

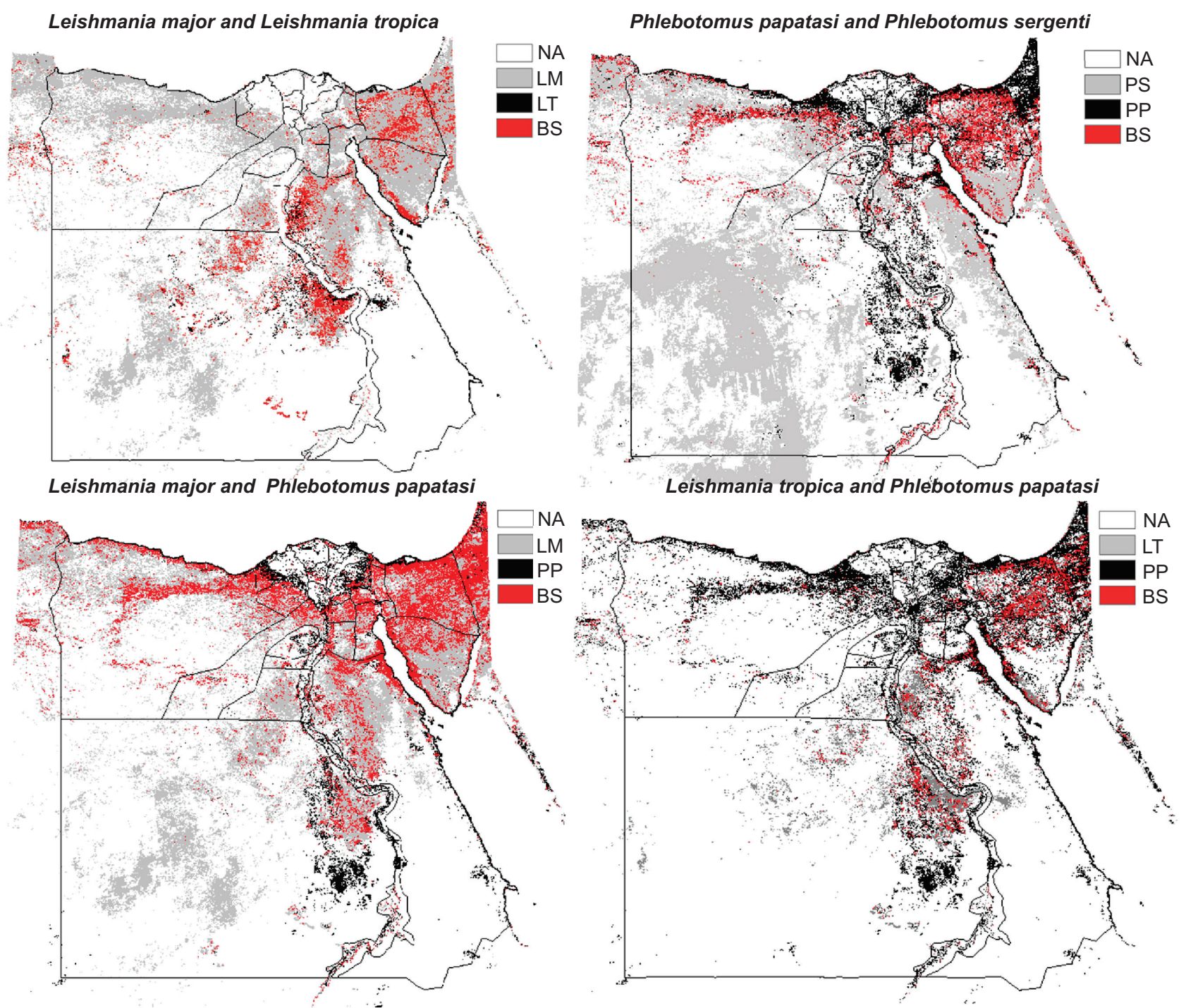

Leishmania major and Phlebotomus sergenti
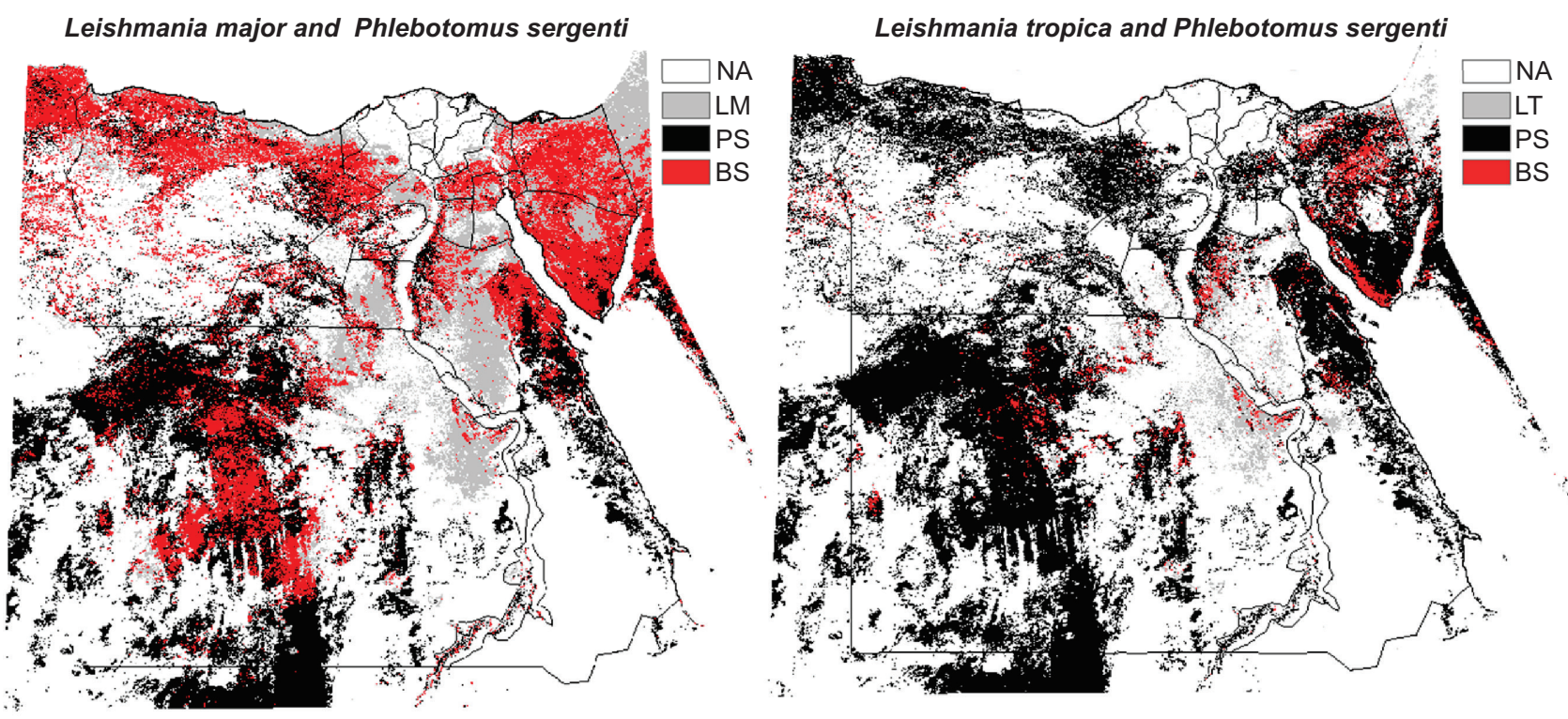

FIGURE 3 - Geographic range overlaps among all combinations of vector-parasite, parasite-parasite, and vector-vector species in leishmaniasis transmission in Egypt. Suitable sites are identified based on presence or absence of each species; presence of Leishmania major (LM), presence of $L$. tropica (LT), presence of Phlebotomus papatasi (PP), presence of P. sergenti (PS), neither of the two species occurs (NA), and both species present (BS). 


\section{ACKNOWLEDGMENTS}

The authors would like to thank the Department of Entomology and the Research and Training Center on Vectors of Diseases of Ain Shams University. Special thanks to Dr. Mohammad Kenawy and Said Doha for their help in the sandfly data collection.

\section{CONFLICT OF INTEREST}

The authors declare that there is no conflict of interest.

\section{FINANCIAL SUPPORT}

The first author is funded by the Graduate Fulbright Egyptian Mission program (EFMP), and the second author was supported by the C-CHANGE IGERT program at the University of Kansas.

\section{REFERENCES}

1. Shehata MG, Samy AM, Doha SA, Fahmy AR, Kaldas RM, Furman BD, et al. First Report of Leishmania tropica from a classical focus of L. major in North-Sinai, Egypt. Am J Trop Med Hyg 2009; 81:213-218.

2. Desjeux P. The increase in risk factors for leishmaniasis worldwide. Trans R Soc Trop Med Hyg 2001; 95:239-243.

3. Alvar J, Velez ID, Bern C, Herrero M, Desjeux P, Cano J, et al. Leishmaniasis worldwide and global estimates of its incidence. PLoS ONE 2012; 7:e35671

4. Wahba MM, Schnur LF, Morsy TA, Merdan A. The characterization of Leishmania major from Phlebotomus papatasi (Scopoli) caught in northern Sinai, Egypt. Trans R Soc Trop Med Hyg 1990; 84:785-786.

5. Fryauff DJ, Modi GB, Mansour NS, Kreutzer RD, Soliman S, Youssef FG. Epidemiology of cutaneous leishmaniasis at a focus monitored by the multinational force and observers in the northeastern Sinai Desert of Egypt. Am J Trop Med Hyg 1993; 49:598-607.

6. Jacobson RL. Leishmania tropica (Kinetoplastida: Trypanosomatidae): a perplexing parasite. Folia Parasitol 2003; 50:241-250.

7. Srinivasan R, Panicker KN, Dhanda V. Population dynamics of Phlebotomus papatasi (Diptera:Phlebotomidae) in Pondicherry, India. Acta Trop 1993; 54:125-130.

8. Orshan L, Szekely D, Khalfa Z, Bitton S. Distribution and seasonality of Phlebotomus sandflies in cutaneous leishmaniasis foci, Judean Desert, Israel. J Med Ent 2010; 47:319-328.

9. Cardenas R, Sandoval CM, Rodríguez-Morales AJ, Franco-Paredes C. Impact of climate variability in the occurrence of leishmaniasis in northeastern Colombia. Am J Trop Med Hyg 2006; 75:273-277.

10. Wasserberg G, Abramsky Z, Kotler BP, Ostfeld RS, Yarom I, Warburg A. Anthropogenic disturbances enhance occurrence of cutaneous leishmaniasis in Israel deserts: patterns and mechanisms. Ecol Appl 2003; 13:868-881.

11. Jeronimo SM, Duggal P, Braz RF, Cheng C, Monteiro GR, Nascimento ET, et al. An emerging peri-urban pattern of infection with Leishmania chagasi, the protozoan causing visceral leishmaniasis in northeast Brazil. Scand J Infect Dis 2004; 36:443-449.

12. Yaghoobi-Ershadi MR, Jafari R, Hanafi-Bojd AA. A new epidemic focus of zoonotic cutaneous leishmaniasis in central Iran. Ann Saudi Med 2004; 24:98-101.

13. Rodriguez-Barraquer I, Gongora R, Prager M, Pacheco R, Montero LM, Navas A, et al. Etiologic agent of an epidemic of cutaneous leishmaniasis in Tolima, Colombia. Am J Trop Med Hyg 2008; 78:276-282.
14. Campbell-Lendrum D, Dujardin JP, Martinez E, Feliciangeli MD, Perez JE, Silans LN, et al. Domestic and peridomestic transmission of American cutaneous leishmaniasis: changing epidemiological patterns present new control opportunities. Mem Inst Oswaldo Cruz 2001; 96:159-162.

15. Peterson AT. Biogeography of diseases: a framework for analysis. Naturwiss 2008; 95:483-491.

16. Peterson AT, Soberón J, Pearson RG, Anderson RP, Martínez-Meyer E, Nakamura M, et al. Ecological Niches and Geographic Distributions. Princeton: Princeton University Press; 2011

17. Soberon J. Grinnellian and Eltonian niches and geographic distributions of species. Ecol Lett 2007; 10:1115-1123.

18. Lawson A. Bayesian Disease Mapping: Hierarchical Modeling in Spatial Epidemiology. New York: Chapman \& Hall; 2008.

19. Cools J, Vanderkimpen P, El Afandi G, Abdelkhalek A, Fockedey S, El Sammany M, et al. An early warning system for flash floods in hyperarid Egypt. Nat Hazards Earth Syst Sci 2012; 12:443-457.

20. Fahmy AR, Samy AM, Doha SA, Shehata MG. Preliminary field investigations on phlebotomine sandflies (Diptera: Psychodidae) from a recent cutaneous leishmaniasis focus in Northern-Sinai, Egypt. Egypt Acad J Biolog Sci 2009; 2:9-15.

21. Barve N, Barve V, Jiménez-Valverde A, Lira-Noriega A, Maher SP, Peterson AT, et al. The crucial role of the accessible area in ecological niche modeling and species distribution modeling. Ecol Mod 2011; 222:1810-1819

22. Lane R. The sand flies of Egypt (Diptera: Phlebotominae). Bull Br Mus Nat Hist Entomol 1986; 52(1):1-35.

23. Wieczorek J, Guo Q, Hijmans R. The point-radius method for georeferencing locality descriptions and calculating associated uncertainty. Int J Geogr Inf Sci 2004; 18:745-767

24. Phillips SJ, Anderson RP, Schapire RE. Maximum entropy modeling of species geographic distributions. Ecol Mod 2006; 190:231-259.

25. Peterson AT, Papeş M, Soberón J. Rethinking receiver operating characteristic analysis applications in ecological niche modeling. Ecol Mod 2008; 213:63-72.

26. Warren DL, Glor RE, Turelli M. Environmental niche equivalency versus conservatism: quantitative approaches to niche evolution. Evolution 2008; 62:2868-2883

27. Maher SP, Ellis C, Gage KL, Enscore RE, Peterson AT. Range-wide determinants of plague distribution in North America. Am J Trop Med Hyg 2010; 83:736-742.

28. Colacicco-Mayhugh MG, Masuoka PM, Grieco JP. Ecological niche model of Phlebotomus alexandri and P. papatasi (Diptera: Psychodidae) in the Middle East. Int J Health Geogr 2010; 9:2.

29. Gonzalez C, Rebollar-Tellez EA, Ibanez-Bernal S, Becker-Fauser I, Martinez-Meyer E, Peterson AT, et al. Current knowledge of Leishmania vectors in Mexico: how geographic distributions of species relate to transmission areas. Am J Trop Med Hyg 2011; 85:839-846.

30. Nimri L, Soubani R, Gramiccia M. Leishmania species and zymodemes isolated from endemic areas of cutaneous leishmaniasis in Jordan. Kinetoplastid Biol Dis 2002; 1:7

31. Jacobson RL, Eisenberger CL, Svobodova M, Baneth G, Sztern J, Carvalho J, et al. Outbreak of cutaneous leishmaniasis in northern Israel. $\mathrm{J}$ Inf Dis 2003; 188:1065-1073.

32. Singer SR, Abramson N, Shoob H, Zaken O, Zentner G, Stein-Zamir C. Ecoepidemiology of cutaneous leishmaniasis outbreak, Israel. Emerg Inf Dis 2008; 14:1424-1426.

33. Azmi K, Nasereddin A, Ereqat S, Schonian G, Abdeen Z. Identification of Old World Leishmania species by PCR-RFLP of the 7 spliced leader RNA gene and reverse dot blot assay. Trop Med Int Health 2010; 15:872880

34. Amro A, Gashout A, Al-Dwibe H, Alam MZ, Annajar B, Hamarsheh O, et al. First molecular epidemiological study of cutaneous leishmaniasis in Libya. PLoS Negl Trop Dis 2012; 6:e1700. 